\title{
Highly Enantioselective [2+2]-Cycloaddition Reactions Catalyzed by a Chiral Aluminum Bromide Complex
}

\author{
Eda Canales and E. J. Corey* \\ Department of Chemistry and Chemical Biology \\ Harvard University, 12 Oxford Street, Cambridge, Massachusetts 02138, USA
}

\section{Supporting Information}

Materials and Methods. Unless stated otherwise, reactions were performed in flame-dried glassware under a positive pressure of nitrogen using freshly distilled dry solvents. Thin-layer chromatography (TLC) was performed using E. Merck silica gel $60 \mathrm{~F}_{254}$ precoated plates $(0.25$ $\mathrm{mm})$. Flash chromatography was performed using Baker silica gel (40 $\mu \mathrm{m}$ particle size). NMR spectra were recorded on Varian Innova-500, or Mercury-400 instruments and calibrated using residual undeuterated solvent as an internal reference. IR spectra were recorded on Avatar 360 FT-IR spectrometer. Low-resolution and high-resolution mass spectral analyses were performed at the Harvard University Mass Spectrometry Center. Analytical high performance liquid chromatography (HPLC) was performed on Isco 2350 Series or Waters 626 HPLC using the indicated chiral column. Gas chromatography (GC) analyses were performed on HewlettPackard 6850 Series GC System equipped with flame ionization detector using a J \& W Scientific Cyclosil-B column (30 m x $0.25 \mathrm{~mm})$ or a $\gamma$-TA column $(30 \mathrm{~m} \times 0.25 \mathrm{~mm})$. Commercial grade reagents and solvents were used without further purification except as indicated below. Dichloromethane was distilled from calcium hydride. 
(S)-(-)-o-Tolyl-oxazaborolidine Precursor of 1. A 100-mL, two-necked, round-bottomed flask equipped with a stir bar, a glass stopper and a $50-\mathrm{mL}$ pressure-equalizing addition funnel (containing a cotton plug and ca. $10 \mathrm{~g}$ of $4 \AA$ molecular sieves, ${ }^{1}$ and functioning as a Soxhlet extractor) fitted on top with a reflux condenser and a nitrogen inlet adaptor was charged with $(S)$ (-)- $\alpha, \alpha$-diphenyl-2-pyrrolidinemethanol $(2.59 \mathrm{~g}, 10.25 \mathrm{mmol}$, from Aldrich or Lancaster), tri-otolylboroxine $^{2}(1.21 \mathrm{~g}, 3.42 \mathrm{mmol})$ and $40 \mathrm{~mL}$ of toluene. The resulting solution was heated to reflux (bath temperature $\sim 145^{\circ} \mathrm{C}$ ). After $3 \mathrm{~h}$, the reaction mixture was cooled to $c a .60{ }^{\circ} \mathrm{C}$ and the addition funnel and condenser were quickly replaced with a short-path distillation head. The mixture was concentrated by distillation (air-cooling) to a volume of $c a .15 \mathrm{~mL}$. This distillation protocol was repeated three times by re-charging with $3 \times 35 \mathrm{~mL}$ of toluene. The solution was then allowed to cool to room temperature and the distillation head was quickly replaced with a vacuum adaptor. Concentration in vacuo (ca. $0.1 \mathrm{mmHg}, 1 \mathrm{~h})$ afforded the corresponding oxazaborolidine as clear oil. A stock solution $(0.25 \mathrm{M})$ of this catalyst precursor was prepared in either toluene or dichloromethane and stored at $-20{ }^{\circ} \mathrm{C}$. A stock solution of (R)-(-)-o-Tolyloxazaborolidine $(0.25 \mathrm{M})$ was prepared from $(R)-(+)-\alpha, \alpha$-diphenyl-2-pyrrolidinemethanol in a similar manner.

Oxazaborolidine-Aluminum Bromide Complex $(\boldsymbol{S})-1$. To an aliquot of the above oxazaborolidine $(0.0625 \mathrm{mmol})$ in dichloromethane $(0.25 \mathrm{~mL})$ at $-40{ }^{\circ} \mathrm{C}$ was added dropwise a solution of aluminum bromide ( $0.05 \mathrm{mmol}, 0.050 \mathrm{~mL}$ of a $1.0 \mathrm{M}$ solution in dibromomethane). A transient strong orange color developed which immediately faded to light yellow. The

1 Molecular sieves (pellets) were dried in vacuo at $c a .200{ }^{\circ} \mathrm{C}$ with a gas burner for $10 \mathrm{~min}$ prior to use.

2 Corey, E. J.; Shibata, T.; Lee, T. W. J. Am. Chem. Soc. 2002, 124, 3808-3809. 
homogeneous catalyst solution of $(S)-\mathbf{1}$ was stirred at $-40{ }^{\circ} \mathrm{C}$ for an additional $30 \mathrm{~min}$. and then used for the [2+2]-cycloaddition reaction. A solution of catalyst $(R)-\mathbf{1}$ was prepared in a similar manner. ${ }^{3}$

\section{Representative Procedure for the use of Catalyst 1 for the Synthesis of 3.}

A solution of $(S)-1(0.05 \mathrm{mmol})$ in dichloromethane-dibromomethane (ca. $0.2 \mathrm{M})$ was cooled to $-78{ }^{\circ} \mathrm{C}$ and 2,2,2-trifluoroethyl acrylate $0.077 \mathrm{~g}, 0.5 \mathrm{mmol}$ ) was added, followed by 1-(tertbutyldimethylsiloxy)cyclohexene $(0.127 \mathrm{~g}, 0.6 \mathrm{mmol})$. The reaction mixture was stirred for $6 \mathrm{~h}$, quenched with $\mathrm{Et}_{3} \mathrm{~N}(100 \mu \mathrm{L})$, and immediately warmed to room temperature. The cloudy solution was directly purified by silica gel column chromatography (elution with $1 \%$ ethyl acetate-hexane) to give the title compound $\mathbf{3}(0.177 \mathrm{mg}, 97 \%)$ as a colorless oil. ${ }^{4}$

\section{Physical Data of the [2+2] Cycloaddition Products:}

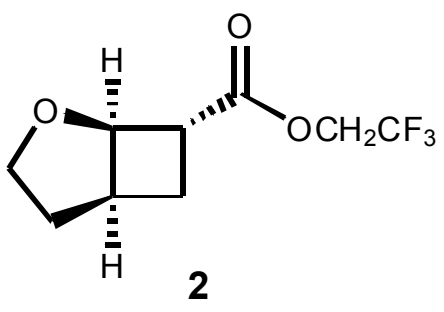

\section{$(1 R, 5 S, 7 R)-2,2,2$-Trifluoroethyl-2-oxa-bicyclo[3.2.0]heptane-7-carboxylate (2).}

A solution of $(S)-1(0.05 \mathrm{mmol})$ in dichloromethane-dibromomethane (ca. $0.2 \mathrm{M})$ was cooled to $-78{ }^{\circ} \mathrm{C}$ and 2,2,2-trifluoroethyl acrylate $(0.154 \mathrm{~g}, 1.0 \mathrm{mmol})$ was added, followed by 2,3dihydrofuran $(0.035 \mathrm{~g}, 0.5 \mathrm{mmol})$. The reaction mixture was stirred for $3 \mathrm{~h}$, quenched with $\mathrm{Et}_{3} \mathrm{~N}$ $(100 \mu \mathrm{L})$, and immediately warmed to room temperature. The cloudy solution was directly

\footnotetext{
${ }^{3}$ Liu, D.; Canales, E.; Corey, E.J. J. Am. Chem. Soc. 2007, 129, 1498-1499.

${ }^{4}$ In large-scale experiments, the chiral ligand diphenylpyrrolidinomethanol is easily recovered in pure condition for reuse. Ryu, D. H.; Lee, T. W.; Corey, E. J. J. Am. Chem. Soc. 2002, 124, 9992-9993.
} 
purified by silica gel column chromatography (elution with $1 \%$ ethyl acetate-hexane) to give the title compound $2(0.097 \mathrm{~g}, 87 \%)$ as a colorless oil. $[\alpha]_{\mathrm{D}}{ }^{25}=-64.0\left(c 1.0, \mathrm{CHCl}_{3}, 99 \%\right.$ ee $) ;{ }^{19} \mathrm{~F}$ NMR $\left(375 \mathrm{MHz}, \mathrm{CDCl}_{3}\right) \delta-74.4 ;{ }^{1} \mathrm{H}$ NMR $\left(400 \mathrm{MHz}, \mathrm{CDCl}_{3}\right) \delta$ 4.70-4.72 (m, 1H), 4.45-4.52 (m, 2H), 4.17-4.22 (m, 1H), 3.93-4.00 (m, 1H), 3.05-3.10 (m, 2H), 2.34-2.42 (m, 1H), 1.58-1.91 (m, $3 \mathrm{H}) ;{ }^{13} \mathrm{C} \mathrm{NMR}\left(100 \mathrm{MHz}, \mathrm{CDCl}_{3}\right)$ 172.5, 123.3 (q, 1C, $\left.J=276 \mathrm{~Hz}\right), 80.22,67.71,60.1$ (q, 1C, $J$ $=36.4 \mathrm{~Hz}$ ), 43.1, 37.1, 31.5, 24.1; IR (film) 2974, 2873, 1753, 1282, 1159, 911, $733 \mathrm{~cm}^{-1}$; HRMS (ESI+) calcd for $\mathrm{C}_{9} \mathrm{H}_{11} \mathrm{~F}_{3} \mathrm{O}_{3}\left(\mathrm{M}+\mathrm{H}^{+}\right)$225.0738, found 225.0737. Enantiomeric purity (99\% ee) was determined by GC analysis using a J \& W Scientific Cyclosil-B column (30 m x $0.25 \mathrm{~mm}, 100^{\circ} \mathrm{C}$ ); retention times: $25.3 \mathrm{~min}$ (major), $26.0 \mathrm{~min}$ (minor).

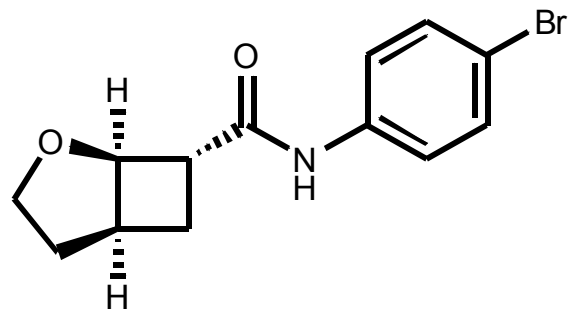

\section{(1R, 5S, 7R)-N-(4-Bromophenyl)-2-oxa-bicyclo[3.2.0] heptane-7-carboxamide:}

Cyclobutane 2 (0.45 g, 2 mmol) was dissolved in $\mathrm{MeOH}: \mathrm{H}_{2} \mathrm{O}$ (2:1) then treated with $\mathrm{LiOH}(0.143$ $\mathrm{g}, 6 \mathrm{mmol}$ ) for $13 \mathrm{~h}$ at ambient temperature and subsequently neutralized with $1 \mathrm{M} \mathrm{HCl}$. The carboxylic acid was extracted from the acidic aqueous phase with $\mathrm{CH}_{2} \mathrm{Cl}_{2}(50 \mathrm{~mL})$, dried over $\mathrm{Na}_{2} \mathrm{SO}_{4}$ for $1 \mathrm{~h}$, filtered, and concentrated. A solution of the crude carboxylic acid was dissolved in dry $\mathrm{CH}_{2} \mathrm{Cl}_{2}(5 \mathrm{~mL}$, ca. $0.4 \mathrm{M})$ and treated with oxalyl chloride $(0.51 \mathrm{~g}, 4 \mathrm{mmol})$. After $1 \mathrm{~h}$ the reaction mixture was concentrated to a viscous yellow oil, diluted with freshly distilled $\mathrm{CH}_{2} \mathrm{Cl}_{2}(10$ $\mathrm{mL}$, ca. $0.2 \mathrm{M})$ and treated with DMAP $(0.24 \mathrm{~g}, 2 \mathrm{mmol})$ followed by 4-bromoaniline (0.68 $\mathrm{g}$ in 4 $\mathrm{mL}$ of $\mathrm{CH}_{2} \mathrm{Cl}_{2}$ ). The reaction mixture was stirred for $16 \mathrm{~h}$ at $\mathrm{rt}$, poured directly into a saturated solution of $\mathrm{NH}_{4} \mathrm{Cl}(100 \mathrm{~mL})$ and extracted with $\mathrm{CH}_{2} \mathrm{Cl}_{2}(100 \mathrm{~mL})$. The combined organic phase 
was washed with $\mathrm{HCl}\left(50 \mathrm{~mL}, 0.1 \mathrm{M}\right.$ aqueous solution), $\mathrm{NaHCO}_{3}(50 \mathrm{~mL}$, saturated solution), brine $(50 \mathrm{~mL})$, dried over $\mathrm{NaSO}_{4}$ and concentrated. The resultant yellow oil was purified by silica gel column chromatography to give the title compound $(0.41 \mathrm{~g}, 70 \%) .[\alpha]_{\mathrm{D}}^{25}=-73.0\left(\mathrm{c} 1.0, \mathrm{CHCl}_{3}\right.$, 99\% ee); m.p. $143-145^{\circ} \mathrm{C}$ (crystallized from $\mathrm{CHCl}_{3}$-hexane, colorless solid); ${ }^{1} \mathrm{H}$ NMR (400 MHz, $\left.\mathrm{CDCl}_{3}\right) \delta 7.66(\mathrm{~s}, 1 \mathrm{H}), 7.39-7.41(\mathrm{~m}, 3 \mathrm{H}), 4.71-4.70(\mathrm{~m}, 1 \mathrm{H}), 4.21(\mathrm{t}, 1 \mathrm{H}, 7.6 \mathrm{~Hz}), 3.95-4.01(\mathrm{~m}$, 1H), 3.05-3.07 (m, 1H), 2.92-2.97 (m, 1H), 2.52-2.59 (m, 1H), 1.86-1.92 (m, 1H), 1.64-1.76(m, $2 \mathrm{H}) ;{ }^{13} \mathrm{C}$ NMR $\left(100 \mathrm{MHz}, \mathrm{CDCl}_{3}\right)$ 171.5, 137.2, 131.8, 121.1, 116.6, 81.1, 67.8, 46.2, 36.8, 31.6, 23.2; IR (film) 3922, 2932, 1652, 1507, 1393, 1156, 1062, 907, 828, $792 \mathrm{~cm}^{-1}$; HRMS (ESI+) calcd for $\mathrm{C} 13 \mathrm{H} 14 \mathrm{BrNO}_{2}\left(\mathrm{M}+\mathrm{H}^{+}\right)$296.0286, found 296.0276. The absolute configuration of this derivative was determined by single crystal x-ray diffraction analysis (see below).

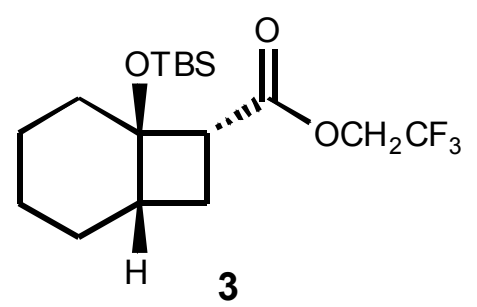

\section{(1S, 6R, 7R)-2,2,2-Trifluoroethyl-6-(tert-Butyldimethylsiloxy)-bicyclo[4.2.0]octane-7-}

carboxylate (3). The crude product was purified by column chromatography elution with $1 \%$ ethyl acetate:hexane afforded $0.089 \mathrm{~g}(97 \%)$ of a colorless oil: Diastereoselectivity (80:20) was determined by ${ }^{1} \mathrm{H}$ NMR spectra analysis of the difference in chemical shift of C7-H : 2.96-3.01 (endoo, major), 3.15-3.19 (exo, minor) and ${ }^{19} \mathrm{~F}$ NMR (375 MHz, $\left.\mathrm{CDCl}_{3}\right) \delta-74.06$ (endo), -74.03 (exo). $[\alpha]_{\mathrm{D}}^{25}=-39.9\left(c 1.0, \mathrm{CHCl}_{3}, 80: 20\right.$ mixture of diastereomers, 92\% ee); ${ }^{1} \mathrm{H}$ NMR (400 $\left.\mathrm{MHz}, \mathrm{CDCl}_{3}\right) \delta$ 4.26-4.56 (m, 2H), 3.15-3.19 (t, 1/5 1H, $\left.J=5.6 \mathrm{~Hz}\right), 2.96-3.01(\mathrm{t}, 4 / 51 \mathrm{H}, J=$ $8.4 \mathrm{~Hz}), 2.49-2.52(\mathrm{~m}, 1 / 5 \mathrm{1H}), 2.27-2.36(\mathrm{~m}, 4 / 51 \mathrm{H}), 1.41-1.83(\mathrm{~m}, 8 \mathrm{H}), 1.22-1.30(\mathrm{~m}, 2 \mathrm{H})$, $0.87(\mathrm{~s}, 4 / 59 \mathrm{H}), 0.83(\mathrm{~s}, 1 / 59 \mathrm{H}), 0.14(\mathrm{~s}, 4 / 53 \mathrm{H}), 0.12(4 / 53 \mathrm{H}), 0.10(\mathrm{~s}, 1 / 53 \mathrm{H}), 0.07(\mathrm{~s}, 1 / 5$ 
$3 \mathrm{H}) ;{ }^{13} \mathrm{C}$ NMR (100 MHz, $\left.\mathrm{CDCl}_{3}\right) \delta 171.0$ (min), 170.6, 123.3 (q, 1C, $J=276 \mathrm{~Hz}$ ), 78.2 (min), 76.4, 60.1 (q, 1C, $J=36.4 \mathrm{~Hz}), 49.60,47.6(\mathrm{~min}), 40.9,40.4(\mathrm{~min}), 31.8,31.6$ (min), 25.5, 21.2, 20.3, 18.7, 17.9, -2.9. IR (film) 2974, 2873, 1753, 1282, 1159, 911, $773 \mathrm{~cm}^{-1}$; LRMS (ESI+): $366.5\left(\mathrm{M}+\mathrm{H}^{+}\right)$. Enantiomeric purity $(92 \%$ ee endo, $0 \%$ ee exo) was determined by $\mathrm{GC}$ analysis using a J \& W Scientific Cyclosil-B column $\left(30 \mathrm{~m}\right.$ x $\left.0.25 \mathrm{~mm}, 100{ }^{\circ} \mathrm{C}\right)$; retention times: 49.2 $\min$ (endo, minor), $50.6 \mathrm{~min}$ (endo, major), $59.3 \mathrm{~min}$ (exo) 61.2 (exo).

\section{Determination of the Absolute Configuration of 3.}

The absolute configuration was determined by the direct conversion of $\mathbf{3}$ to the known alcohol $(1 R, 6 S, 8 S)$-1-tert-butyldimethylsiloxy-8-hydroxymethylbicyclo [4.2.0]octane. A solution of 3 (0.089 g, $0.24 \mathrm{mmol})$ in $\mathrm{CH}_{2} \mathrm{Cl}_{2}(1 \mathrm{~mL})$ was treated with DIBAL $(0.72 \mathrm{mmol}, 1 \mathrm{M}$ solution in hexane) at $-78{ }^{\circ} \mathrm{C}$ then immediate dry-ice bath removal and continuous stirring at ambient temperature. After $1.5 \mathrm{~h}$, the reaction flask was cooled to $-78^{\circ} \mathrm{C}$ followed by dropwise addition of $\mathrm{MeOH}(1 \mathrm{~mL})$ then immediate dry-ice bath removal and continuous stirring at ambient temperature for $2 \mathrm{~h}$. The resulting white precipitate was filtered through Celite, concentrated and the clear oil residue was purified by silica gel column chromatography ( $5 \%$ ethyl acetate-hexane) to give the known endo compound (1R, $6 S$, $8 S)$-1-tert-butyldimethylsiloxy-8hydroxymethylbicyclo[4.2.0]octane as the major product. The and spectral data were identical to those previously reported for this compound; $[\alpha]_{\mathrm{D}}{ }^{23}=-25.2\left(c 0.3, \mathrm{CHCl}_{3}\right) .^{5}$

\footnotetext{
${ }^{5}$ Takasu, K.; Nagao, S.; Ueno, M.; Ihara, M. Tetrahedron 2004, 60, 2071-2078.
} 


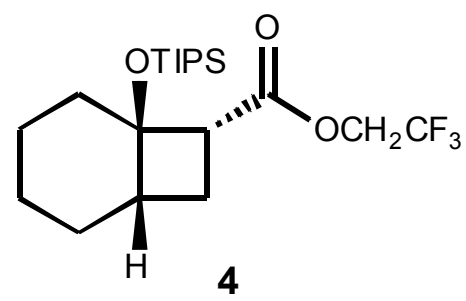

$(1 S, 6 R, 7 R)-2,2,2$-Trifluoro-ethyl-6-(triisopropylsiloxy)-bicyclo[4.2.0]octane-7-carboxylate

(4). The crude product was purified by column chromatography elution with $1 \%$ ethyl acetatehexane afforded $0.101 \mathrm{~g}(99 \%)$ of a colorless oil. Diastereoselectivity (97:3) was determined by the ${ }^{1} \mathrm{H}$ NMR spectra analysis of the difference in chemical shift of C7-H: 3.00-3.05 (endo, major), 3.27-3.31 (exo, minor) and ${ }^{19} \mathrm{~F}$ NMR (375 MHz, $\left.\mathrm{CDCl}_{3}\right) \delta-74.04$ (endo), -74.31 (exo). $[\alpha]_{\mathrm{D}}{ }^{25}=-38.3\left(c 1.0, \mathrm{CHCl}_{3}, 92 \%\right.$ ee); ${ }^{1} \mathrm{H}$ NMR $\left(400 \mathrm{MHz}, \mathrm{CDCl}_{3}\right) \delta 4.42-4.48(\mathrm{~m}, 2 \mathrm{H}), 3.00-$ $3.05(\mathrm{t}, 1 \mathrm{H}, J=8.0 \mathrm{~Hz}), 2.31-2.37(\mathrm{~m}, 1 \mathrm{H}), 1.76-1.83(\mathrm{~m}, 1 \mathrm{H}), 1.42-1.72(\mathrm{~m}, 7 \mathrm{H}), 1.25-1.34(\mathrm{~m}$, 2H), $1.07(\mathrm{~s}, 21 \mathrm{H}) ;{ }^{13} \mathrm{C} \mathrm{NMR}\left(100 \mathrm{MHz}, \mathrm{CDCl}_{3}\right) \delta 170.7,123.3(\mathrm{q}, 1 \mathrm{C}, J=276 \mathrm{~Hz}), 76.2,60.0$ (q, $1 \mathrm{C}, J=36.4 \mathrm{~Hz}$ ), 50.0, 41.4, 32.4, 23.4, 21.3, 20.5, 18.6, 18.16, 13.1; IR (film) 2940, 2866, 1750, 1280, 1160, 1132, 881, $676 \mathrm{~cm}^{-1}$; HRMS (ESI+) calcd for $\mathrm{C}_{20} \mathrm{H}_{35} \mathrm{~F}_{3} \mathrm{O}_{3} \mathrm{Si}\left(\mathrm{MH}^{+}\right)$409.2386, found 409.2380. Enantiomeric purity (92\% ee) was determined by reduction with DIBAL to the corresponding alcohol, conversion to the (R)-MTPA ester derivative and ${ }^{1} \mathrm{H}$ NMR analysis (500 $\left.\mathrm{MHz}, \mathrm{CDCl}_{3}\right): \delta 4.48$ (d, 1H, major), 4.45 (d, 1H, major), $4.23(\mathrm{~d}, 1 \mathrm{H}$, minor), 4.40 (d, 1H, minor). 


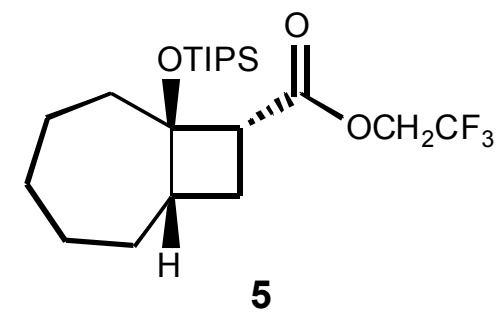

$(1 S, 7 R, 8 R)$ - 2,2,2-Trifluoroethyl-7-(triisopropylsiloxy)-bicyclo[5.2.0]nonane-8-carboxylate

(5). The crude product was purified by column chromatography elution with $1 \%$ EtOAc-hexane afforded $0.104 \mathrm{~g}(99 \%)$ of a colorless oil: Diastereoselectivity (99:1) was determined by ${ }^{1} \mathrm{H}$ NMR spectra analysis of the difference in chemical shift of the C8-H: 3.16-3.21 (endo), 3.28$3.36($ exo $)$ and ${ }^{19} \mathrm{~F}$ NMR $\left(375 \mathrm{MHz}, \mathrm{CDCl}_{3}\right) \delta-73.94($ endo) $),-74.01($ exo $) .[\alpha]_{\mathrm{D}}{ }^{25}=-39.2(c 1.0$, $\mathrm{CHCl}_{3}, 99 \%$ ee). ${ }^{1} \mathrm{H}$ NMR (400 MHz, $\left.\mathrm{CDCl}_{3}\right) \delta$ 4.41-4.50 (m, 2H), 3.16-3.21 (t, $1 \mathrm{H}, J=9.2 \mathrm{~Hz}$ ), 2.43-2.39 (m, 1H), 2.13-2.05 (m, 1H), 1.95-1.90 (m, 1H), 1.80-1.56 (m, 10H), $1.09(\mathrm{~s}, 21 \mathrm{H}) ;{ }^{13} \mathrm{C}$ $\operatorname{NMR}\left(100 \mathrm{MHz}, \mathrm{CDCl}_{3}\right) \delta 170.8,123.3(\mathrm{q}, 1 \mathrm{C}, J=276 \mathrm{~Hz}), 82.2,60.1$ (q, 1C, $\left.J=36.4 \mathrm{~Hz}\right), 48.6$, 47.7, 34.4, 33.9, 31.9, 31.6, 26.6, 23.6, 18.2, 13.3. . IR (film) 2924, 2866, 1750, 1279, 1161, 1124, 881, 674, $650 \mathrm{~cm}^{-1}$; LRMS (ESI+): $423.6\left(\mathrm{M}+\mathrm{H}^{+}\right)$. Enantiomeric purity (99\% ee) was determined by reduction with DIBAL to the corresponding alcohol, conversion to the $(R)$-MTPA ester derivative and ${ }^{1} \mathrm{H}$ NMR integration $\left(400 \mathrm{MHz}, \mathrm{CDCl}_{3}\right)$ : $\delta 4.49$ (d, 1H, major), $4.46(\mathrm{~d}, 1 \mathrm{H}$, major), 4.44 (d, 1H, minor), 4.40 (d, 1H, minor).

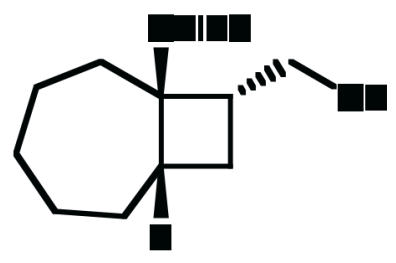

$((1 S, 7 R, 8 R)$-7-(triisopropylsiloxy)-bicyclo[5.2.0]non-8-yl) methanol . A solution of $\mathbf{5}(0.86$ g, $2.0 \mathrm{mmol})$ in $\mathrm{CH}_{2} \mathrm{Cl}_{2}(1 \mathrm{~mL})$ was treated with DIBAL $(6.0 \mathrm{mmol}, 1 \mathrm{M}$ solution in hexane $)$ at $78{ }^{\circ} \mathrm{C}$ then immediate dry-ice bath removal and continuous stirring at ambient temperature. 
After $1.5 \mathrm{~h}$, the reaction flask was cooled to $-78^{\circ} \mathrm{C}$ followed by dropwise addition of $\mathrm{MeOH}(4$ $\mathrm{mL}$ ) then immediate dry-ice bath removal and continuous stirring at ambient temperature for $2 \mathrm{~h}$. The resulting white precipitate was filtered through Celite, concentrated and the clear oil residue was purified by silica gel column chromatography ( $5 \%$ ethyl acetate-hexane) to give the title compound $(0.52 \mathrm{~g}, 80 \%$ yield $) .[\alpha]_{\mathrm{D}}{ }^{25}=-12.0\left(c\right.$ 1.0, $\mathrm{CHCl}_{3}, 99 \%$ ee); ${ }^{1} \mathrm{H}$ NMR (400 MHz, $\left.\mathrm{CDCl}_{3}\right)$ S 3.75-3.78 (dd, $\left.1 \mathrm{H}, \mathrm{J}=5.5,11.0 \mathrm{~Hz}\right), 3.53-3.57(\mathrm{dd}, 1 \mathrm{H}, J=8.5,11.0 \mathrm{~Hz}), 2.39-2.43(\mathrm{~m}$, $1 \mathrm{H}), 2.28-2.32(\mathrm{~m}, 1 \mathrm{H}), 2.08-2.14(\mathrm{~m}, 1 \mathrm{H}), 1.79-1.95(\mathrm{~m}, 3 \mathrm{H}), 1.53-1.70(\mathrm{~m}, 4 \mathrm{H}), 1.12-1.29(\mathrm{~m}$, 4H), $1.08(\mathrm{~m}, 21 \mathrm{H}), 0.86-0.92(\mathrm{~m}, 1 \mathrm{H}) ;{ }^{13} \mathrm{C} \mathrm{NMR}\left(100 \mathrm{MHz}, \mathrm{CDCl}_{3}\right) \delta$ 80.7, 63.5, 47.2, 46.4, 34.9, 32.6, 32.3, 26.7, 24.5, 23.6, 18.4, 13.5; IR (film) 3307, 2919, 2863, 1436, 1134, 1082, $1010,880,669 \mathrm{~cm}^{-1}$.

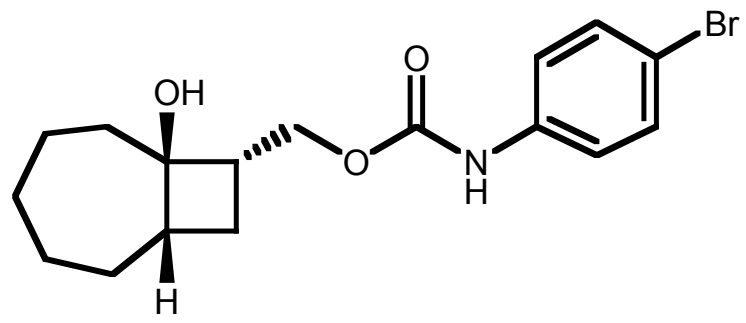

\section{$((1 S, 7 R, 8 S)$-7-hydroxybicyclo[5.2.0]nonan-8-yl) methyl 4-bromophenyl carbamate.}

A solution of $((1 S, 7 R, 8 R)-7$-(triisopropylsiloxy)-bicyclo[5.2.0]non-8-yl) methanol (0.52 g, 1.6 mmol) in THF (3.2 mL, ca. $0.5 \mathrm{M})$ was treated with TBAF ( $8.0 \mathrm{mmol}$ of a1 M solution in THF) at ambient temperature then refluxed for $8 \mathrm{~h}$. Upon cooling, the solution was poured into a saturated $\mathrm{NH}_{4} \mathrm{Cl}$ solution, extracted with ethylacetate, dried over $\mathrm{NaSO}_{4}$, and concentrated. The crude product was then taken up in benzene (ca. $\sim 0.5 \mathrm{M}$ ) and 4-bromophenyl isocyanate $(0.297 \mathrm{~g}$, $1.5 \mathrm{mmol})$ was added followed by triethylamine $(0.20 \mathrm{~g}, 2.0 \mathrm{mmol})$. After $0.5 \mathrm{~h}$ at $\mathrm{rt}$, the reaction mixture was purified by silica gel column chromatography, $10 \%$ to $20 \%$ ethylacetatehexane to give the title compound $(0.37 \mathrm{~g}, 62 \%$ yield $)$. $[\alpha]_{\mathrm{D}}{ }^{25}=-12.0(c 1.0$, acetone $) ; \mathrm{mp}=115-$ 
$117{ }^{\circ} \mathrm{C}$ (crystallized from acetone-hexane, clear solid); ${ }^{1} \mathrm{H}$ NMR (400 MHz, $\mathrm{d}_{6}$-acetone) $\delta 8.65$ (bs, 1H), 7.55-7.60 (d, 2H), 7.41-7.50 (d, 2H), 4.10-4.13 (m, 2H), 3.81 (s, 1H), 2.40-2.45 (m, 1H), 2.10-2.14 (m, 1H), 1.45-2.12 (m, 10H), 1.19-1.23 (m, 1H), 0.86-0.92 (m, 1H); ${ }^{13} \mathrm{C}$ NMR $(100$ MHz, $\mathrm{d}_{6}$-acetone) $\delta 154.3,139.7,132.3,120.7,114.9,78.4,65.3,47.2,44.8,35.4,32.9,31.5$, 30.4, , 27.5, 24.2; IR (film) 3283, 2914, 2856, 1699, 1594, 152, 1224, 1066, $811 \mathrm{~cm}^{-1}$; HRMS $(\mathrm{ESI}+)$ calcd for $\mathrm{C} 13 \mathrm{H} 14 \mathrm{BrNO}_{2}\left(\mathrm{MH}^{+}(-\mathrm{OH})\right)$ 351.0834, found 351.0738. The absolute configuration of this derivative was determined by single crystal x-ray diffraction analysis (see below).

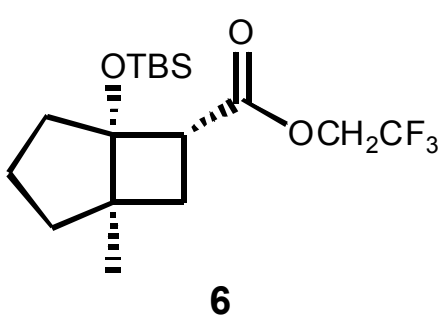

$(1 R, 5 R, 6 R)-2,2,2-$ Trifluoroethyl- 5 -(tert-Butyldimethylsiloxy)-1-methylbicyclo[3.2.0] heptane-6-carboxylate (6). A solution of $(S)$-1 $(0.05 \mathrm{mmol})$ in dichloromethanedibromomethane (ca. $0.2 \mathrm{M}$ ) was cooled to $-78{ }^{\circ} \mathrm{C}$ and 2,2,2-trifluoroethyl acrylate $(0.077 \mathrm{~g}, 0.5$ mmol) was added, followed by followed by 1-(tert-butyldimethylsiloxy)-2-methyl-cyclopentene $(0.127 \mathrm{~g}, 0.6 \mathrm{mmol})$. The reaction mixture was stirred for $3 \mathrm{~h}$, quenched with $\mathrm{Et}_{3} \mathrm{~N}(100 \mu \mathrm{L})$, and immediately warmed to room temperature. The cloudy solution was directly purified by silica gel column chromatography (elution with $1 \%$ ethyl acetate-hexane) to give the title compound $\mathbf{6}$ $(0.181 \mathrm{~g}, 99 \%)$ as a colorless oil. Diastereoselectivity (1:99) was determined by ${ }^{1} \mathrm{H}$ NMR spectra analysis of the difference in chemical shift of C6-H: 2.95-2.99 (exo, major), and ${ }^{19} \mathrm{~F}$ NMR (375 $\left.\mathrm{MHz}, \mathrm{CDCl}_{3}\right) \delta-74.00 .{ }^{1} \mathrm{H}$ NMR $\left(400 \mathrm{MHz}, \mathrm{CDCl}_{3}\right) \delta 4.56-4.65(\mathrm{~m}, 1 \mathrm{H}), 4.16-4.26(\mathrm{~m}, 1 \mathrm{H})$, 2.95-2.99 (t, 1H, $J=9.2 \mathrm{~Hz}), 2.11-2.16(\mathrm{~m}, 1 \mathrm{H}), 1.93-1.97(\mathrm{~m}, 1 \mathrm{H}), 1.57-1.83(\mathrm{~m}, 6 \mathrm{H}), 0.85(\mathrm{~s}$, 
9H), 0.08 (s, 3H), 0.05 (s, 3H); ${ }^{13} \mathrm{C}$ NMR (100 MHz, $\left.\mathrm{CDCl}_{3}\right) \delta 171.0,123.3$ (q, $\left.1 \mathrm{C}, J=276 \mathrm{~Hz}\right)$, 85.1, 60.4 (q, 1C, $J=36.4 \mathrm{~Hz}), 47.4,46.8,40.4,39.3,27.9,25.7,22.6,21.7,18.2,-2.1,-2.7$. IR (film) 2931, 2857, 1756, 1281, 1104, 888, 835, $773 \mathrm{~cm}^{-1}$; HRMS (ESI+) calcd for $\mathrm{C}_{17} \mathrm{H}_{29} \mathrm{~F}_{3} \mathrm{O}_{3} \mathrm{Si}$ $\left(\mathrm{MH}^{+}\right) 367.1916$ found 367.1915. Enantiomeric purity (98\% ee) was determined by reduction with DIBAL to the corresponding alcohol and GC analysis using a J \& W Scientific Cyclosil-B column (30 m x $\left.0.25 \mathrm{~mm}, 100{ }^{\circ} \mathrm{C}\right)$; retention times: $60.1 \mathrm{~min}$ (minor) and $61.3 \mathrm{~min}$ (major). Absolute configuration was determined by conversion to the known compound 5H-Indene-5-one1,2,3,6,7-7a-hexahydro-7a-methyl- $(R)(\mathbf{1 0})$ see below.

\section{Determination of the Relative Configuration of 6.}

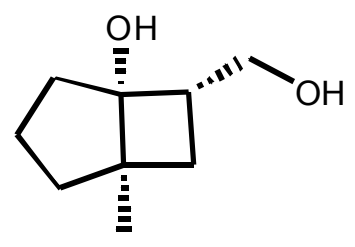

\section{$(1 R, 5 R, 7 S)-7-($ hydroxymethyl)-5-methylbicyclo[3.2.0]heptane-1-ol.}

A solution of $6(0.724 \mathrm{~g}, 1.0 \mathrm{mmol})$ in $\mathrm{CH}_{2} \mathrm{Cl}_{2}(1 \mathrm{~mL})$ was treated with DIBAL $(3 \mathrm{mmol}, 1 \mathrm{M}$ solution in hexane) at $-78{ }^{\circ} \mathrm{C}$ then immediate dry-ice bath removal and continuous stirring at ambient temperature. After $1.5 \mathrm{~h}$, the reaction flask was cooled to $-78^{\circ} \mathrm{C}$ followed by dropwise addition of $\mathrm{MeOH}(1 \mathrm{~mL})$ then immediate dry-ice bath removal and continuous stirring at ambient temperature for $2 \mathrm{~h}$. The resulting white precipitate was then filtered through celite and concentrated. To a solution of the crude alcohol $(1.0 \mathrm{mmol})$ in THF $(3.2 \mathrm{~mL}$, ca. $0.5 \mathrm{M})$ was treated with TBAF (5.0 mmol of a1 M solution in THF) at ambient temperature then refluxed for $8 \mathrm{~h}$. Upon cooling, the solution was poured into a saturated $\mathrm{NH}_{4} \mathrm{Cl}$ solution, extracted with ethylacetate, dried over $\mathrm{NaSO}_{4}$, and concentrated. The resulting diol was purified by flash silica 
gel chromatography $50 \%$ ethyl acetate-hexane to give the title compound $(0.093 \mathrm{~g}, 60 \%)$. $[\alpha]_{\mathrm{D}}{ }^{25}$ $=+4.9\left(c 1.0, \mathrm{CHCl}_{3}, 99 \%\right.$ ee $) ;{ }^{1} \mathrm{H}$ NMR $\left(400 \mathrm{MHz}, \mathrm{CDCl}_{3}\right) \delta 3.72-3.83(\mathrm{~m}, 2 \mathrm{H}), 2.68(\mathrm{~b}, 1 \mathrm{H})$, 1.84-1.88 (m, 1H), 1.32-1.62 (m, 7H), 1.21-1.30, (m, 1H), 1.09 (s, 3H); ${ }^{13} \mathrm{C}$ NMR $(100 \mathrm{MHz}$ $\mathrm{CDCl}_{3}$ ) $883.5,63.5,45.3,41.7,41.5,39.8,28.8,22.3,20.5$; IR (film) 3355, 2926, 2850, 1437, 1309, 1172, 1094, 1054, 1031, $1003 \mathrm{~cm}^{-1}$; LRMS (ESI+) $157.1\left(\mathrm{M}+\mathrm{H}^{+}\right)$.

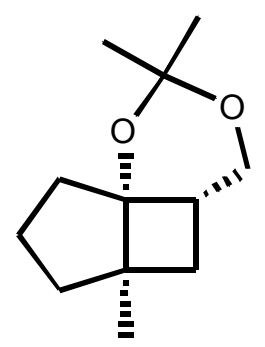

\section{$(1 R, 6 S, 8 R)-3,3,8$-trimethyl-2,4-dioxotricyclo $\left[6.3 .0 .0^{1,6}\right]$ nonane.}

A solution $(1 R, 5 R, 7 S)$-7-(hydroxymethyl)-5-methylbicyclo[3.2.0]heptane-1-ol $(0.047 \mathrm{~g}, 0.3$ mmol) in DMF (0.6 mL, ca. $0.5 \mathrm{M}$ ) was treated with TsOH (cat.), dimethyoxypropane (0.093 g, $0.9 \mathrm{mmol}$ ) and refluxed for $8 \mathrm{~h}$. The reaction mixture was poured into a saturated solution of $\mathrm{NaHCO}_{3}(50 \mathrm{~mL})$, and extracted with ether $(30 \mathrm{~mL})$. The ethereal layer was subsequently washed with brine $(50 \mathrm{~mL})$, dried over $\mathrm{Na}_{2} \mathrm{SO}_{4}$ and concentrated. Silica gel purification $(2 \%$, ethyl acetate-hexane) afforded the desired acetal $(0.042 \mathrm{~g}, 70 \%$ yield $) .[\alpha]_{\mathrm{D}}^{25}=+7.8(c 1.0$, $\left.\mathrm{CHCl}_{3}\right) ;{ }^{1} \mathrm{H}$ NMR $\left(400 \mathrm{MHz}, \mathrm{CDCl}_{3}\right) \delta 3.91(\mathrm{dd}, 1 \mathrm{H}, J=11.2,6.8 \mathrm{~Hz}), 3.59(\mathrm{dd}, 1 \mathrm{H}, J=11.2$, $7.2 \mathrm{~Hz}), 1.79-1.48(\mathrm{~m}, 6 \mathrm{H}), 1.90-1.84(\mathrm{~m}, 2 \mathrm{H}), 2.10-2.06(\mathrm{~m}, 1 \mathrm{H}), 1.40(\mathrm{~s}, 3 \mathrm{H}), 1.37(\mathrm{~s}, 3 \mathrm{H})$ 1.13 (s, 3H); ${ }^{13} \mathrm{C}$ NMR (100 MHz, $\left.\mathrm{CDCl}_{3}\right) \delta$ 97.6, 81.0 62.8, 45.9, 40.5, 33.1, 32.6, 27.9, 26.3, 22.6, 22.4, 20.7; IR (film) 2926, 1367, 1222, 1147, 1105, 1062, 1036, $864 \mathrm{~cm}^{-1}$; LRMS (ESI+) $197.1\left(\mathrm{M}+\mathrm{H}^{+}\right)$. 


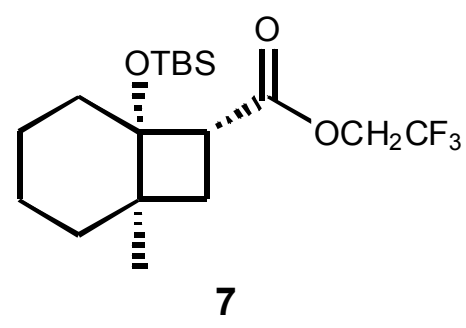

$(1 R, 6 R, 7 R)-2,2,2$-Trifluoroethyl - 6 -(tert-Butyldimethylsiloxy)-1-methylbicyclo[4.2.0] octane -7-carboxylate (7). The crude product was purified by column chromatography (elution with $1 \%$ ethyl acetate-hexane) afforded $0.094 \mathrm{~g}$ (99\%). Diastereoselectivity (10:90) was determined by ${ }^{1} \mathrm{H}$ NMR spectra analysis of the difference in chemical shift of C7-H: 3.23 (exo), $2.98($ endo $)$ and ${ }^{19} \mathrm{~F}$ NMR $\left(375 \mathrm{MHz}, \mathrm{CDCl}_{3}\right) \delta-73.8($ exo $),-73.9($ endo $) .[\alpha]_{\mathrm{D}}{ }^{25}=-19.5(c 1.0$, $\mathrm{CHCl}_{3}, 98 \%$ ee major isomer); ${ }^{1} \mathrm{H}$ NMR (400 MHz, $\left.\mathrm{CDCl}_{3}\right) \delta 4.43-4.51(\mathrm{~m}, 2 \mathrm{H}), 3.23(\mathrm{t}, 1 \mathrm{H}, J$ $=9.6 \mathrm{~Hz}), 1.81-1.89(\mathrm{~m}, 1 \mathrm{H}), 1.20-1.79(\mathrm{~m}, 10 \mathrm{H}), 1.09(\mathrm{~s}, 3 \mathrm{H}), 0.89(\mathrm{~s}, 9 \mathrm{H}), 0.14(\mathrm{~s}, 3 \mathrm{H}), 0.09(\mathrm{~s}$, $3 \mathrm{H}) ;{ }^{13} \mathrm{C}$ NMR $\left(100 \mathrm{MHz}, \mathrm{CDCl}_{3}\right) \delta 171.5,123.3$ (q, 1C, $\left.J=276 \mathrm{~Hz}\right), 77.6,60.4(\mathrm{q}, 1 \mathrm{C}, J=36.4$ Hz), 48.2, 41.5, 33.1, 32.0, 26.8, 25.7, 24.6, 21.5, 20.0, 18.4, -3.0, -3.5; IR (film) 2930, 2858, 1750, 1280, 1162, 1097, 834, $774 \mathrm{~cm}^{-1}$; LRMS (ESI+) $381.5\left(\mathrm{M}+\mathrm{H}^{+}\right)$. Enantiomeric purity $(98 \%$ ee) was determined was determined by reduction with DIBAL to the corresponding alcohol and GC analysis using a $\mathrm{J} \& \mathrm{~W}$ Scientific Cyclosil-B column $\left(30 \mathrm{~m}\right.$ x $\left.0.25 \mathrm{~mm}, 135{ }^{\circ} \mathrm{C}\right)$; retention times: 68.5 min (major) and 71.8 min (minor). Absolute configuration determined by conversion to the known compound [2(3H)-Napthalenone, 4,4a,5,6,7,8-hexahydro-4a-methyl, $(R)$-] (11) see below. 


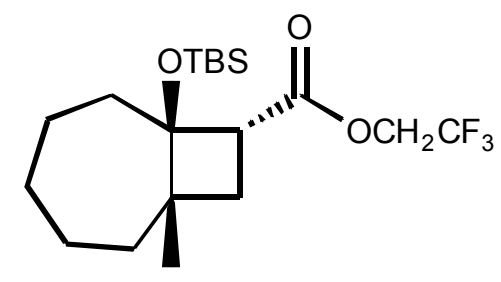

8

$(1 S, 7 S, \quad 8 R)-\quad 2,2,2$-Trifluoroethyl- 7 -(tert-Butyldimethylsiloxy)-1-methylbicyclo[5.2.0] nonane-8-carboxylate (8). The crude product was purified by column chromatography (elution with $1 \%$ ethyl acetate-hexane) afforded $0.096 \mathrm{~g}(97 \%) .{ }^{19} \mathrm{~F}$ NMR $\left(375 \mathrm{MHz}, \mathrm{CDCl}_{3}\right) \delta-73.7$ (exo), -73.9 (endo). $[\alpha]_{\mathrm{D}}{ }^{25}=-20.7$ (c 1.0, $\mathrm{CHCl}_{3}, 98 \%$ ee major isomer); ${ }^{1} \mathrm{H} \mathrm{NMR}(400 \mathrm{MHz}$, $\left.\mathrm{CDCl}_{3}\right) \delta$ 4.41-4.54 (m, 2H), 3.23-3.28 (t, $\left.1 \mathrm{H}, J=10 \mathrm{~Hz}\right), 1.18-1.26(\mathrm{~m}, 10 \mathrm{H}), 1.21-1.31(\mathrm{~m}$, 2H), 1.13 (s, 3H), $0.92(\mathrm{~s}, 9 \mathrm{H}), 0.19$ (s, 3H), 0.07 (s, 3H); $\left.{ }^{13} \mathrm{C} \mathrm{NMR} \mathrm{(100} \mathrm{MHz,} \mathrm{CDCl}_{3}\right) \delta$ 171.3, $123.3(\mathrm{q}, 1 \mathrm{C}, J=276 \mathrm{~Hz}), 82.3,60.4(\mathrm{q}, 1 \mathrm{C}, J=36.4 \mathrm{~Hz}), 46.7,46.0,42.5,33.8,31.7,31.6,31.0$, 26.0, 24.5, 23.7, 18.7, -3.3, -3.5; IR (film) 2928, 2856, 1750, 1279, 1168, 830, $773 \mathrm{~cm}^{-1}$; LRMS $(\mathrm{ESI}+) 395.2\left(\mathrm{M}+\mathrm{H}^{+}\right)$. Enantiomeric purity and absolute configuration was determined by conversion to the known compound 1,11-dehydo-10-oxo-7-methyl-bicyclo[5.4.0]undecane (see below) and GC analysis using $\gamma$-TA column $\left(30 \mathrm{~m} \times 0.25 \mathrm{~mm}, 150^{\circ} \mathrm{C}\right)$; retention times: $18.0 \mathrm{~min}$ (minor) and $18.8 \mathrm{~min}$ (major).

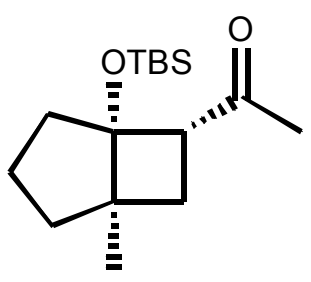

$(1 R, 5 R, 6 R)$-5-(tert-Butyldimethylsiloxy)-1-methylbicyclo[3.2.0]heptane-6-methyl ketone.

A slurry of $6(0.165 \mathrm{~g}, 0.45 \mathrm{mmol})$ and $N, O$-dimethylhydroxyamine hydrochloride $(0.132 \mathrm{~g}$, $1.36 \mathrm{mmol})$ in THF $(2.3 \mathrm{~mL})$ was cooled to $-30{ }^{\circ} \mathrm{C}$ and treated with an ethereal solution of 
$\operatorname{MeMgBr}(2.74 \mathrm{mmol} ; 3.0 \mathrm{M})$ then stirred for $1 \mathrm{~h}$. Clean formation of the Weinreb amide was observed by TLC along with small amounts of ketone product. To this solution a second portion of $\mathrm{MeMgBr}$ was added $(1.35 \mathrm{mmol})$ and the reaction flask was slowly warmed to $0{ }^{\circ} \mathrm{C}$. After 5 hours complete consumption of the Weinreb amide was observed by TLC. The reaction mixture was poured into a saturated solution of $\mathrm{NH}_{4} \mathrm{Cl}(100 \mathrm{~mL})$, extracted with ether $(50 \mathrm{~mL})$, the combined ethereal portions were dried over sodium sulfate, and concentrated. The ${ }^{1} \mathrm{H}$ NMR of the crude material revealed $<2 \%$ of the undesired alcohol. The resulting clear oil was purified by silica gel column chromatography with $1 \%$ ether-pentane to give the title compound (108 mg, $85 \%) .[\alpha]_{\mathrm{D}}^{20}=-30.0\left(c 1.0, \mathrm{CHCl}_{3}\right) ;{ }^{1} \mathrm{H} \mathrm{NMR}\left(400 \mathrm{MHz}, \mathrm{CDCl}_{3}\right) \delta 2.96(\mathrm{t}, 1 \mathrm{H}, J=7.2 \mathrm{~Hz}), 2.20-$ $2.25(\mathrm{~m}, 1 \mathrm{H}), 2.08(\mathrm{~s}, 3 \mathrm{H}), 1.94-1.98(\mathrm{~m}, 1 \mathrm{H}), 1.59-.1 .82(\mathrm{~m}, 3 \mathrm{H}), 1.18-1.36(\mathrm{~m}, 3 \mathrm{H}), 1.03(\mathrm{~s}$ 3H), 0.83 (s, 9H), 0.07(s, 3H), 0.05 (s, 3H); $\left.{ }^{13} \mathrm{C} \mathrm{NMR} \mathrm{(100} \mathrm{MHz,} \mathrm{CDCl}_{3}\right) \delta$ 206.3, 85.9, 53.4, $46.7,40.9,39.3,34.1,31.6,25.9,22.6,21.9,18.2,-2.14,-2.24$; IR (film) 2952, 2928, 2857, 1716, 1254, 1192, 1104, 1081, 881, 834, 772, $679 \mathrm{~cm}^{-1}$; LRMS (ESI+) $283.3\left(\mathrm{M}+\mathrm{H}^{+}\right)$.

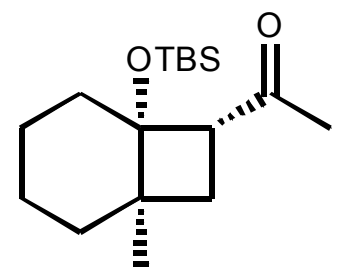

$(1 R, 6 R, 7 R)-6$-(tert-Butyldimethylsiloxy)-1-methylbicyclo[4.2.0]heptane-7-methyl ketone. A slurry of $7(0.097 \mathrm{~g}, 0.25 \mathrm{mmol})$ and $\mathrm{N}$, O-dimethylhydroxyamine hydrochloride $(0.076 \mathrm{~g}$, $0.77 \mathrm{mmol})$ in THF $(1.25 \mathrm{~mL})$ was cooled to $-30{ }^{\circ} \mathrm{C}$ and treated with an ethereal solution of $\mathrm{MeMgBr}(1.54 \mathrm{mmol} ; 3.0 \mathrm{M})$ then stirred for $3 \mathrm{~h}$. Clean formation of the Weinreb amide was observed by TLC along with small amounts of ketone product. To this solution a second portion of $\mathrm{MeMgBr}$ was added $(0.75 \mathrm{mmol})$ and the reaction flask was slowly warmed to $0{ }^{\circ} \mathrm{C}$. After 16 hours complete consumption of the Weinreb amide was observed by TLC. The reaction mixture 
was poured into a saturated solution of $\mathrm{NH}_{4} \mathrm{Cl}(100 \mathrm{~mL})$, extracted with ether $(50 \mathrm{~mL})$, the combined ethereal portions were dried over sodium sulfate, and concentrated. The resulting clear oil was purified by silica gel column chromatography with $1 \%$ ether-pentane to give the title compound (55 mg major isomer and $6 \mathrm{mg}$ of the minor isomer $82 \%$ combined yield). The major isomer was fully characterized: $[\alpha]_{\mathrm{D}}{ }^{20}=-39.4\left(c 1.0, \mathrm{CHCl}_{3}\right) ;{ }^{1} \mathrm{H} \mathrm{NMR}\left(400 \mathrm{MHz}, \mathrm{CDCl}_{3}\right)$ $\delta 3.21(\mathrm{t}, 1 \mathrm{H}, J=7.6 \mathrm{~Hz}), 2.23(\mathrm{t}, 1 \mathrm{H}, J=10 \mathrm{~Hz}), 2.05-2.08(\mathrm{~m}, 1 \mathrm{H}), 2.07(\mathrm{~s}, 3 \mathrm{H}), 1.68-1.79(\mathrm{~m}$, 2H), 1.38-1.60 (m, 6H), $0.96(\mathrm{~s}, 3 \mathrm{H}), 0.85(\mathrm{~s}, 9 \mathrm{H}), 0.11(\mathrm{~s}, 3 \mathrm{H}), 0.09(\mathrm{~s}, 3 \mathrm{H}) ;{ }^{13} \mathrm{C} \mathrm{NMR}(100 \mathrm{MHz}$, $\left.\mathrm{CDCl}_{3}\right) \delta 207.4,84.4,50.7,40.9,38.5,34.6,31.8,30.2,25.9,23.7,23.31,21.4,14.0,-1.18,-1.71$; IR (film) 2926, 2854, 1701, 1463, 1357, 1252, 1090, 1055, 829, 771, $669 \mathrm{~cm}^{-1}$; LRMS (ESI+) $297.3\left(\mathrm{M}+\mathrm{H}^{+}\right)$.

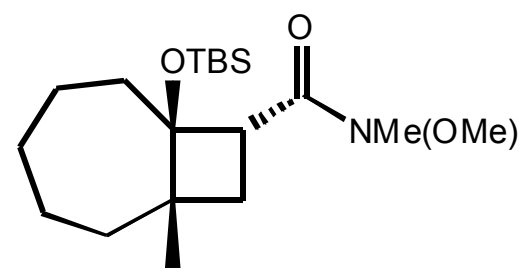

\section{$(1 S, \quad 7 S, \quad 8 R)-7-($ tert-Butyldimethylsiloxy)-1-methylbicyclo[5.2.0] nonane-8- $N$-methoxy- $N$ -} methyl carboxamide. A slurry of $8(0.098 \mathrm{~g}, 0.25 \mathrm{mmol})$ and $N, O$-dimethylhydroxyamine hydrochloride $(0.073 \mathrm{~g}, 0.75 \mathrm{mmol})$ in THF $(1.25 \mathrm{~mL})$ was cooled to $-30{ }^{\circ} \mathrm{C}$ and treated with an ethereal solution of $\mathrm{MeMgBr}(1.5 \mathrm{mmol} ; 3.0 \mathrm{M})$ then stirred for $1 \mathrm{~h}$. Clean formation of the Weinreb amide was observed by TLC. The reaction mixture was quenched with saturated solution of ammonium chloride and extracted (3 X $20 \mathrm{~mL}$ of ether). The reaction mixture was poured into a saturated solution of $\mathrm{NH}_{4} \mathrm{Cl}(100 \mathrm{~mL})$, extracted with ether $(50 \mathrm{~mL})$, the combined ethereal portions were dried over sodium sulfate, and concentrated. The resulting clear oil was purified by silica gel column chromatography with $1 \%$ ether-pentane to give the title compound $(80 \mathrm{mg}, 91 \%) .[\alpha]_{\mathrm{D}}{ }^{20}=-20.8\left(c 1.0, \mathrm{CHCl}_{3}\right) ;{ }^{1} \mathrm{H} \mathrm{NMR}\left(400 \mathrm{MHz}, \mathrm{CDCl}_{3}\right) \delta 3.63$ 
(s, 3H), 3.41-3.49(m, 1H), 3.17 (s, 3H), 2.02-2.08 (m, 1H), 1.85-1.95 (m, 1H), 1.45-1.77 (m, 9H), $1.14(\mathrm{~s}, 3 \mathrm{H}), 0.91(\mathrm{~s}, 9 \mathrm{H}), 0.08(\mathrm{~s}, 3 \mathrm{H}), 0.07$ (s, 3H); ${ }^{13} \mathrm{C} \mathrm{NMR}\left(100 \mathrm{MHz}, \mathrm{CDCl}_{3}\right) \delta$ 164.4, 82.9, $61.0,46.1,43.5,42.0,34.0,31.6,31.5,26.1,24.72,23.9,23.6,22.6,18.7,-3.0,-3.4 ;$ IR (film) 2926, 2854, 1655, 1463, 1252, 1168, 1087, 831, $773 \mathrm{~cm}^{-1}$; LRMS (ESI+) $342.2\left(\mathrm{M}+\mathrm{H}^{+}\right)$.

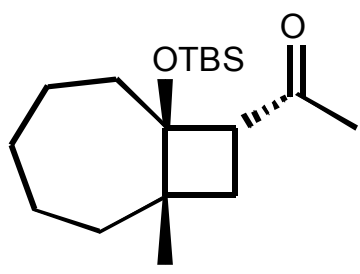

\section{$(1 S, 7 S, 8 R)$-7-(tert-Butyldimethylsiloxy)-1-methylbicyclo[5.2.0]nonane-8-methyl ketone.}

A solution of $(1 S, 7 S, 8 R)$-7-(tert-Butyldimethylsiloxy)-1-methylbicyclo[5.2.0]nonane-8- $N$ methoxy- $N$-methyl carboxamide $(0.071 \mathrm{~g}, 0.20 \mathrm{mmol})$ in THF $(1 \mathrm{~mL})$ was cooled to $-30{ }^{\circ} \mathrm{C}$ and treated with an ethereal solution of $\mathrm{MeLi}(0.6 \mathrm{mmol} ; 1.6 \mathrm{M})$ then stirred for $16 \mathrm{~h}$. The reaction mixture was poured into a saturated solution of $\mathrm{NH}_{4} \mathrm{Cl}(50 \mathrm{~mL})$, extracted with ether $(30 \mathrm{~mL})$, the combined ethereal portions were dried over sodium sulfate, and concentrated. The ${ }^{1} \mathrm{H} N M R$ of the crude material revealed $5 \%$ of unreacted Weinreb amide. The resulting clear oil was purified by silica gel column chromatography with $1 \%$ ether-pentane to give the title compound $(0.044 \mathrm{~g}, 72 \%) \cdot[\alpha]_{\mathrm{D}}{ }^{20}=-36.7\left(c 1.0, \mathrm{CHCl}_{3}\right) ;{ }^{1} \mathrm{H} \mathrm{NMR}\left(400 \mathrm{MHz}, \mathrm{CDCl}_{3}\right) \delta 3.19(\mathrm{t}, 1 \mathrm{H}, J=10$ $\mathrm{Hz}), 2.11(\mathrm{~s}, 3 \mathrm{H}), 1.45-1.81(\mathrm{~m}, 12 \mathrm{H}), 1.11(\mathrm{~s}, 3 \mathrm{H}), 0.92(\mathrm{~s}, 9 \mathrm{H}), 0.15(\mathrm{~s}, 3 \mathrm{H}), 0.13(\mathrm{~s}, 3 \mathrm{H}) ;{ }^{13} \mathrm{C}$ NMR (100 MHz, $\left.\mathrm{CDCl}_{3}\right) \delta$ 209.1, 83.2, 54.3, 45.2, 42.3, 33.7, 31.7, 31.6, 30.5, 26.1, 25.8, 24.5, $23.7,18.8,-2.8,-2.9$; IR (film) 2926, 2854, 1700, 1463, 1253, 1089, 1056, 830, $773 \mathrm{~cm}^{-1}$; HRMS (ESI+) calcd for $\mathrm{C}_{18} \mathrm{H}_{34} \mathrm{O}_{2} \mathrm{Si}\left(\mathrm{MH}^{+}\right)$311.2328, found 311.2393. 


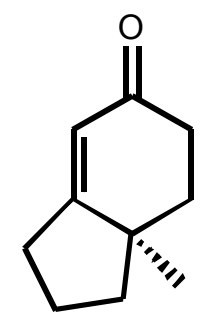

\section{H-Indene-5-one-1,2,3,6,7-7a-hexahydro-7a-methyl-(R) (10).}

A solution of $(1 S, 5 R, 6 R)-5$-(tert-Butyldimethylsiloxy)-1-methylbicyclo[3.2.0]heptane-6-methyl ketone $(0.080 \mathrm{~g}, 0.28 \mathrm{mmol})$ in $\mathrm{THF}(\mathrm{ca} .0 .2 \mathrm{M})$ was cooled to $0{ }^{\circ} \mathrm{C}$ and treated with $\mathrm{TBAF}(2.0$ $\mathrm{mmol}, 1.0 \mathrm{M}$ in $\mathrm{THF})$. After $0.5 \mathrm{~h}$ the reaction flask was brought slowly to ambient temperature, stirred for an addition $0.5 \mathrm{~h}$, concentrated, dissolved in a solution of $5 \% \mathrm{NaOH}$ in methanol (5 $\mathrm{mL}$ ), and refluxed for $16 \mathrm{~h}$. The reaction mixture was neutralized with $0.1 \mathrm{M} \mathrm{HCl}$, concentrated and purified by silica gel chromatography $10 \%$ ether-pentane to give the known title compound $(0.033 \mathrm{~g}, 80 \%)$. The spectral data were identical to those previously reported for this compound. $[\alpha]_{\mathrm{D}}{ }^{20}=-94.0\left(c\right.$ 1.0, EtOH); ${ }^{1} \mathrm{H}$ NMR $\left(400 \mathrm{MHz}, \mathrm{CDCl}_{3}\right) \delta 5.75(\mathrm{~s}, 1 \mathrm{H}), 2.28-2.78(\mathrm{~m}, 4 \mathrm{H})$, 1.98-2.02 (m, 2H), 1.40-1.55 (m, 2H), $1.15(\mathrm{~s}, 3 \mathrm{H})$. The absolute configuration follows from the sign of rotation. ${ }^{6}$

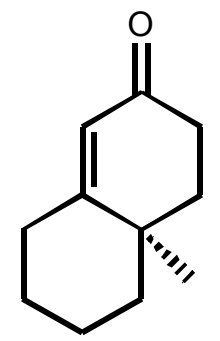

\section{[2(3H)-Napthalenone, 4,4a,5,6,7,8-hexahydro-4a-methyl, $(R)-](11)$.}

A solution of $(1 S, 6 R, 7 R)$-6-(tert-Butyldimethylsiloxy)-1-methylbicyclo[3.2.0]heptane-7-methyl ketone $(0.030 \mathrm{~g}, 0.1 \mathrm{mmol})$ in $\mathrm{THF}(\mathrm{ca} .0 .2 \mathrm{M})$ was cooled to $0{ }^{\circ} \mathrm{C}$ and treated with $\mathrm{TBAF}(1.0$ $\mathrm{mmol}, 1.0 \mathrm{M}$ in THF). After $0.5 \mathrm{~h}$ the reaction flask was brought slowly to ambient temperature,

\footnotetext{
${ }^{6}$ Pfau, M.; Revial, G.; Guigant, A.; Angelo, J. J. Am. Chem. Soc., 1985, 107, 273-274.
} 
stirred for an addition $0.5 \mathrm{~h}$, concentrated, dissolved in a solution of $5 \% \mathrm{NaOH}$ in methanol $(5$ $\mathrm{mL}$ ), and stirred for $3 \mathrm{~h}$. The reaction mixture was neutralized with $0.1 \mathrm{M} \mathrm{HCl}$, concentrated and purified by silica gel chromatography $10 \%$ ethyl acetate-hexane to give the known title compound $(0.014 \mathrm{~g}, 87 \%)$. The spectral data were identical to those previously reported for this compound. $[\alpha]_{\mathrm{D}}{ }^{20}=-203.0(c$ 1.0, EtOH $) ;{ }^{1} \mathrm{H}$ NMR $\left(400 \mathrm{MHz}, \mathrm{CDCl}_{3}\right) \delta 5.76(\mathrm{~s}, 1 \mathrm{H}), 2.51-2.48$ (m, 1H), 2.28-2.30 (m, 1H), 2.21-2.37 (m, 2H), 1.31-1.88 (m, 8H), $1.21(\mathrm{~s}, 3 \mathrm{H})$. The absolute configuration follows from the sign of rotation. ${ }^{7}$

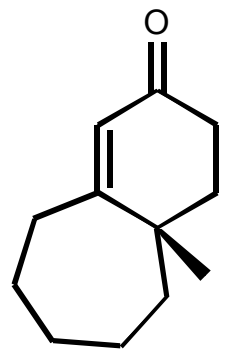

\section{(S)-1,11-dehydro-10-oxo-7-methyl-bicyclo[5.4.0] undecane (12).}

A solution of $(1 S, 6 R, 8 R)$-7-(tert-Butyldimethylsiloxy)-1-methylbicyclo[3.2.0]heptane-8-methyl ketone $(0.031 \mathrm{~g}, 0.1 \mathrm{mmol})$ in $\operatorname{THF}(\mathrm{ca} .0 .2 \mathrm{M})$ was cooled to $0{ }^{\circ} \mathrm{C}$ and treated with TBAF $(1.0$ $\mathrm{mmol}, 1.0 \mathrm{M}$ in THF). After $0.5 \mathrm{~h}$ the reaction flask was brought slowly to ambient temperature, stirred for an addition $0.5 \mathrm{~h}$, concentrated, dissolved in a solution of $5 \% \mathrm{NaOH}$ in methanol $(5$ $\mathrm{mL}$ ), and heated to $40{ }^{\circ} \mathrm{C}$ for $3 \mathrm{~h}$. The reaction mixture was neutralized with $0.1 \mathrm{M} \mathrm{HCl}$, concentrated and purified by silica gel chromatography $10 \%$ ethyl acetate-hexane to give the known title compound $(0.016 \mathrm{~g}, 87 \%$ yield). The spectral data were identical to those previously reported for this compound: $[\alpha]_{\mathrm{D}}^{20}=+172(c 1.0, \mathrm{EtOH}) .{ }^{8}{ }^{1} \mathrm{H} \mathrm{NMR}\left(400 \mathrm{MHz}, \mathrm{CDCl}_{3}\right) \delta 5.82(\mathrm{~s}$, $1 \mathrm{H}), 2.51-2.53(\mathrm{~m}, 1 \mathrm{H}), 2.29-2.40(\mathrm{~m}, 1 \mathrm{H}), 2.20-2.26(\mathrm{~m}, 1 \mathrm{H}), 1.21-2.20(\mathrm{~m}, 11 \mathrm{H}), 1.17(\mathrm{~s}, 3 \mathrm{H})$.

\footnotetext{
${ }^{7}$ Doering, W. E.; Birladeanu, L.; Sarma, K.; Shao, L. S. J. Am. Chem. Soc. 1996, 118, 6660-6665

${ }^{8}$ Goubaud, V.; Azerad, R. Synth. Comm. 1996, 26, 915-922.
} 
The absolute configuration was confirmed by single crystal x-ray diffraction analysis of the thiosemicarbazone (see below).

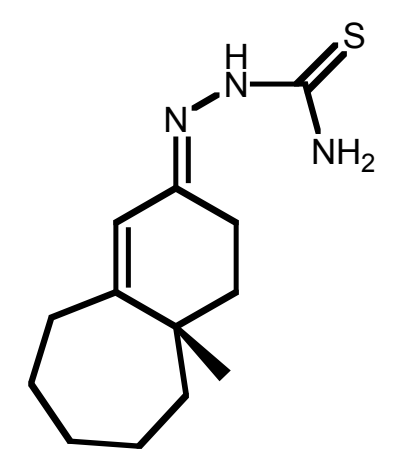

\section{$(S, E)$-2-(4a-methyl-3,4,4a,5,6,7,8,9-octahydrobenzo[7]annulen-2-one) thiosemivarbazone.}

A solution of $12(0.089 \mathrm{~g}, 0.5 \mathrm{mmol})$ in absolute ethanol $(10 \mathrm{~mL})$ was treated with thiosemicarbazide $(0.055 \mathrm{~g}, 0.6 \mathrm{mmol})$, TFA (cat. amount) and stirred at ambient temperature for $2 \mathrm{~h}$. The reaction mixture was poured into a saturated solution of $\mathrm{NaHCO}_{3}(40 \mathrm{~mL})$ and extracted with $\mathrm{CH}_{2} \mathrm{Cl}_{2}(30 \mathrm{~mL})$. The combined organic phase was dried over $\mathrm{Na}_{2} \mathrm{SO}_{4}$, concentrated, and the resulting yellow oil was purified by silica gel column chromatography with $5 \%$ ethyl acetate-hexane to give the title compound $(0.113 \mathrm{~g}, 90 \%)$. $[\alpha]_{\mathrm{D}}{ }^{25}=+285\left(c 0.7, \mathrm{CHCl}_{3}\right)$; $\mathrm{mp}=160-161{ }^{\circ} \mathrm{C}$ (crystallized from ethyl acetate-hexane, clear solid); ${ }^{1} \mathrm{H}$ NMR $(400 \mathrm{MHz}$, CDCl3) $\delta 8.63$ (bs, 1H), 7.24 (bs, 1H), 6.29 (bs, 1H), $5.87(\mathrm{~s}, 1 \mathrm{H}), 2.53-2.58(\mathrm{~m}, 1 \mathrm{H}), 2.11-2.37$ (m, 3H), 1.74-1.99 (m, 10H), $1.03(\mathrm{~s}, 3 \mathrm{H}) ;{ }^{13} \mathrm{C}$ NMR (100 MHz, $\left.\mathrm{CDCl}_{3}\right)$ 8178.4, 162.3, 123.4, 41.1, 38.3, 35.5, 33.8, 33.6, 31.2, 30.8, 26.0, 23.6, 20.9; IR (film) 3422, 3240, 3145, 2921, 2851, 1577, 1490, 1282, 1075, $863 \mathrm{~cm}^{-1}$; HRMS (ESI+) calcd for $\mathrm{C}_{13} \mathrm{H}_{21} \mathrm{~N}_{3} \mathrm{~S}\left(\mathrm{MH}^{+}\right) 252.1534$, found 252.1535 . 